\title{
Multiple pulmonary hydatid cysts
}

\author{
J. V. Deshmane \\ B.Sc., M.S. \\ Lecturer in Surgery, \\ Medical College Hospital, Nagpur, India
}

THE LUNG is the second commonest site in man, where the intermediate larval-cysticercal stage of Echinococcus granulosus develops. It is not unusual to find more than one cyst in the lung in man. However, the successful removal of more than two cysts from the lung is exceptionally rare. The incidence of hydatids occurring in lungs varies in different countries (Spencer, 1962), viz:

Greece 30\% (Toole et al., 1953)

Australia $25 \%$

France 9\% (Barrett)

New Zealand $40 \%$

A survey of hydatid disease in the Waikato Hospital Board area from 1942 to 1959 (New Zealand) by Ellis Dick (1962-63) showed that 124 cases were treated and the distribution among them was as follows:

liver-55

lung -49

lung and liver combined-10

However, the incidence of multiple pulmonary hydatid cysts was rarely mentioned. d'Abreu \& Lambert Rogers (1938) reported the first case of two cysts, one on each side of the lung. Borrie (1962-63), in his report of fifty cases of thoracic hydatid disease, mentions that only seven cases of multiple cysts were seen. The maximum number of cysts was only two, in the seven cases seen. Barrett (1960) reports that the largest number of primary hydatid cysts in the lung was three. One of the three was a simple cyst and the remaining two were ruptured in the bronchial tree and the patient died. Thomas (1965) reports seeing three cysts in the lungs. Deve (Barrett, 1960) analysed thirty-one cases of multiple metastatic hydatid cysts in the lungs and found that the primary cyst was in the heart (twenty cases), liver (eight cases) and iliac bones (three cases). Thus the successful removal of three cysts or more from the lung is very exceptional. The following is a report of three cases of pulmonary hydatid cysts, successfully operated upon, where the number of cysts removed was more than two in each case.

\section{Case 1}

Mr S., a 40-year-old male, had, at the time of admission, two cysts in the right lung (upper and lower lobe one each), four cysts in the left lung (both lobes involved) and two cysts in the liver. The two cysts on the right side were unequal in size. However, all the cysts on the left side and one on the right (upper lobe) appeared to be almost equal in size, signifying that they were all of almost the same age (Fig. 1).

It was decided to do the right thoracotomy first in order to release the right lung, the lessaffected side (10 September 1963). Both cysts were removed and ruptured during removal. The patient went into anaphylactic shock but soon recovered.

The second operation (29 October 1963), a laparotomy by a Rutherford Morrison incision on the right side, consisted of marsupialization of a large cyst in the liver. The second cyst, a smaller one, $1 \frac{1}{2} \times 1 \frac{1}{2}$ in., was just attached to the free margin of the liver and was therefore excised with a portion of the liver. Haemostasis was achieved

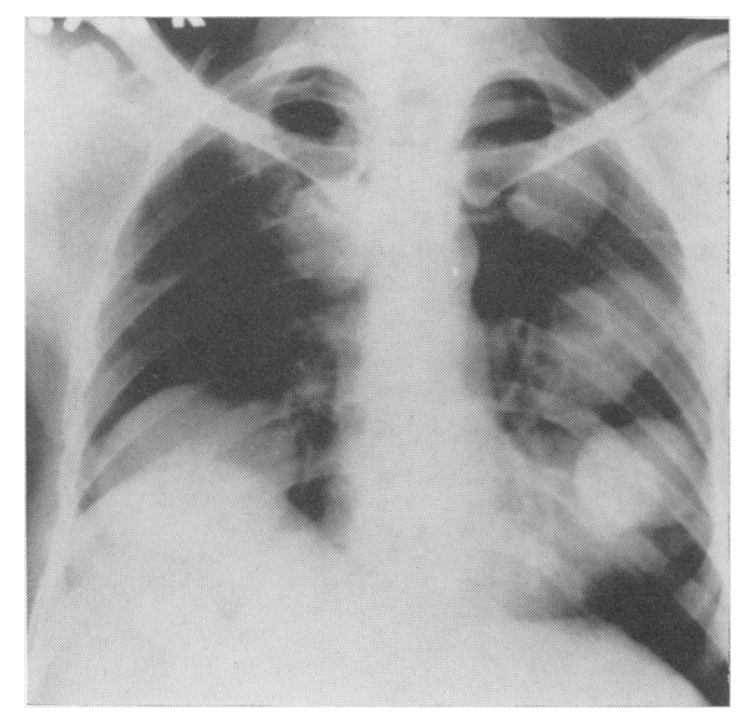

FIG. 1. 
by suturing the cut margins of the liver together. The patient again had anaphylactic shock but he soon recovered.

The third operation (10 January 1964) was a left thoracotomy and all the four cysts were removed. Two were ruptured during removal and the remaining two were excised intact (Fig. 2).

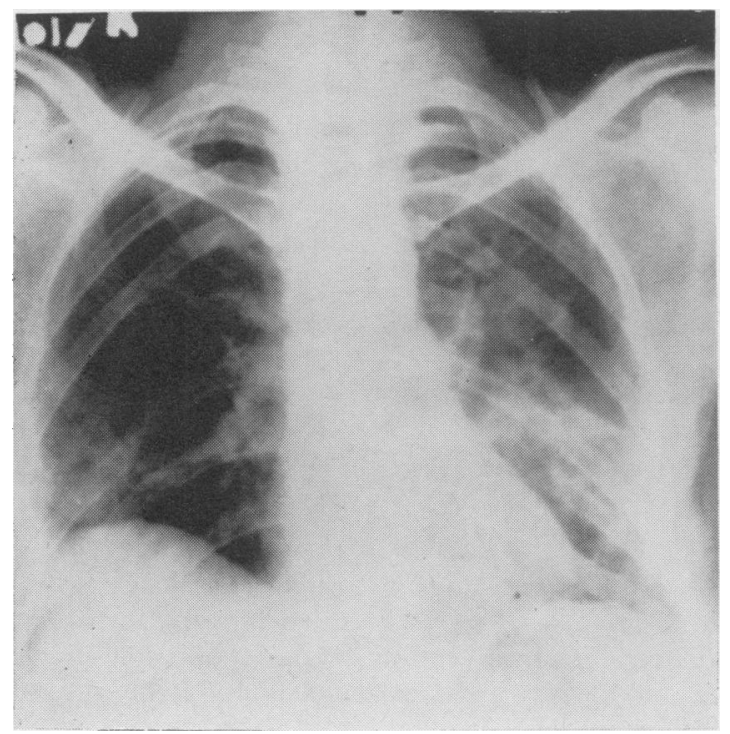

FIG. 2.

Barrett's technique (Barrett \& Thomas, 195253), employing a bag to receive the cyst was not used during the thoracic operations. The lobe of the lung containing the cyst was isolated by arranging packs, soaked in $5 \%$ formalin, on all sides. The pericyst was incised to expose the whitish shining ectocyst and the removal was effected by alternate blunt and sharp dissection to isolate the ectocyst from the surrounding pericyst formed by the lung tissue. Formalin was not injected into the lung cyst-a procedure which the writer always employs in dealing with hydatid cysts of the liver. The final stage in the removal of the cyst was assisted by the anaesthetist, who employed positive pressure ventilation to express it. Obliteration of the pulmonary cavity was performed by inserting several purse-string sutures. Although the patient had anaphylactic shock during each operation the severity was diminished with each successive procedure. The rest of the postoperative management was uneventful.

In this patient, the bilateral involvement of the lung and the different sizes of the cysts, suggest that they were of different ages. Either one was primary (the larger in the right lower lobe) and the remaining cysts represented either a complicated liver cyst or a complicated primary lung cyst, or all of the pulmonary cysts could be due to a complicated liver cyst but developing at intervals. The same argument applies to the two different-sized cysts occurring in the liver.

\section{Case 2}

Mr P., a 35-year-old male, was admitted with three cysts in the right lung, all of different sizes. One of the cysts appeared to have ruptured and showed a fluid level with a perivesicular pneumocyst (Figs. 3 and 4).

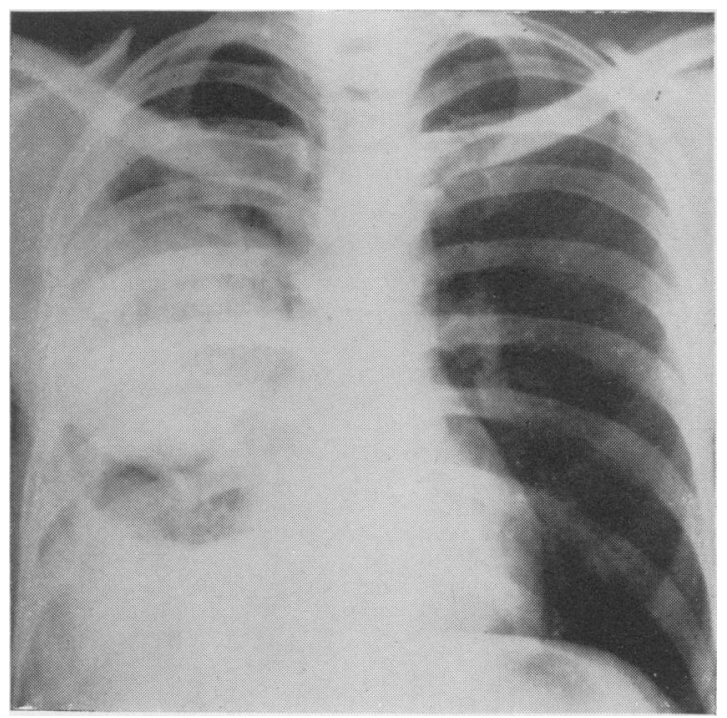

Fig. 3.

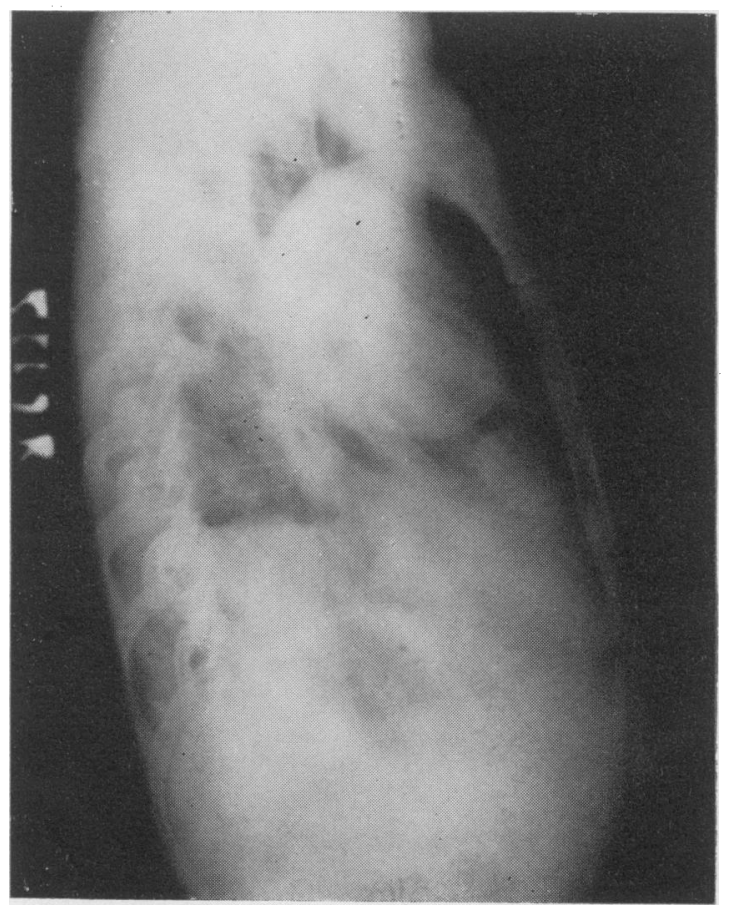

Fig. 4. 
A right thoracotomy was done on 14 January 1964. There were dense adhesions between the lung and pleura. During their division the infected large cyst was ruptured. On completing evacuation of the ectocyst, the distinction between the three lobes was seen to be completely lost and the lung was grossly destroyed. Ultimately, a pneumonectomy was performed. This patient also had anaphylactic shock during the operation, and subsequently developed a post-pneumonectomy empyema which was successfully treated by repeated aspirations and antibiotics.

\section{Case 3}

Miss C., a 28-year-old female, was admitted with three hydatid cysts of different sizes in the right lung (Fig. 5). Removal of all the three cysts with obliteration of the cyst cavities was carried out. She had an uneventful postoperative period (Fig. 6).

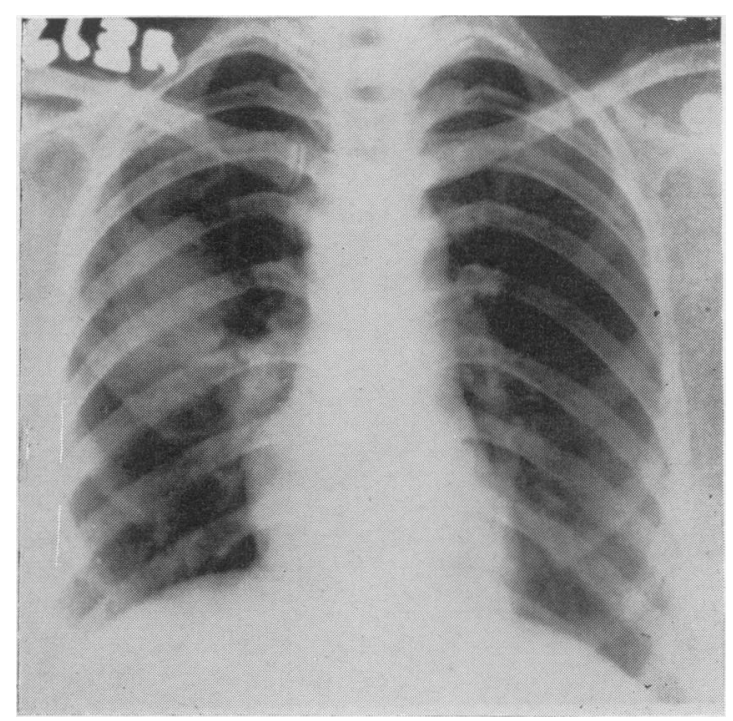

Fig. 5.

\section{Clinical picture}

All of the three patients complained of cough with breathlessness and expectoration, pain in the chest and a painful mass in the abdomen (Case 1) and haemoptysis. The duration of symptoms was 6 months to 18 months. Blood examination revealed a moderate rise in eosinophils (10$21 \%$ ) and Casoni's test was positive in the first two cases, while it was negative in case 3 . The complement fixation test could not be done in these patients.

\section{Past association with dogs}

Case 1 was a shepherd and had a number of dogs and sheep. Case 2 also gave a positive history

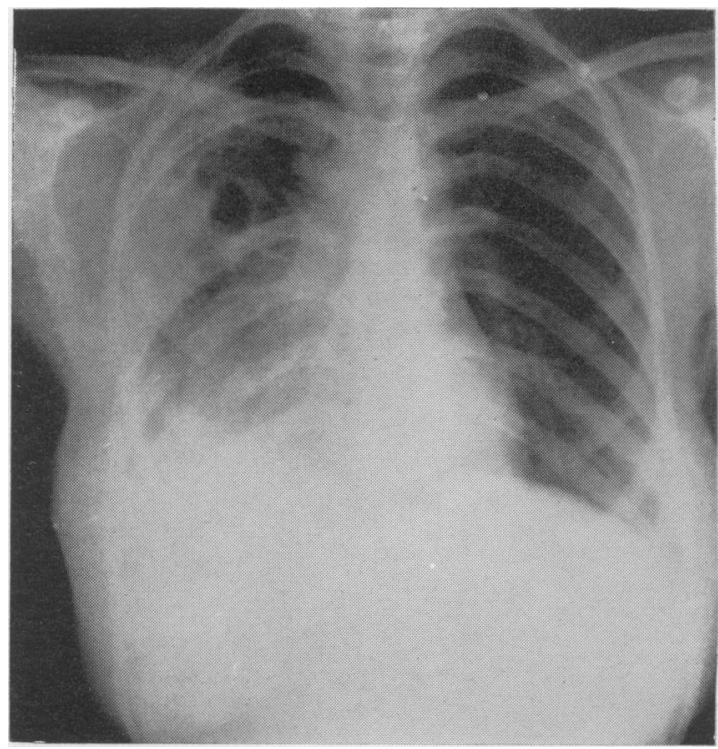

Fig. 6.

of contact with dogs. No such history was a vailable in Case 3. None of them had had any kind of surgical intervention before admission. Cases 2 and 3 had no clinical evidence of hydatid cysts anywhere else in the body (liver, bone, heart, etc.).

\section{Result and prognosis}

All three patients were completely relieved. The prognosis in hydatid disease is excellent. The expansion of the lung postoperatively, with obliteration of the cavity, was complete.

\section{The significance of multiple cysts in the lung}

As the pathogenesis of pulmonary hydatid is well known, it is not intended to describe it further. According to Barrett (1960) multiple hydatids in the chest grow as a result of:

(a) Multiple primary infestations from one or more dogs on one or more occasions.

(b) Complications that befall a simple lungcyst such as partial or complete rupture into the bronchial tree or as a result of imperfect surgery.

(c) Leakage from a primary hydatid cyst present elsewhere in the body into a vein, commonly from the liver, heart or iliac bones. The genesis of multiple unilateral cysts is slightly different from multiple bilateral cysts. Multiple unilateral cysts may be due to complications that befall a simple cyst in the lung, i.e. rupture, or to complicated liver cysts settling in the lung parenchyma, either through the diaphragm (adhesions produced, fistula formed), or by blood spread.

Multiple bilateral hydatids grow because of 
embolic hydatid infection in the blood vessels, spread through the lymphatics, or possibly from an endobronchial spread from one lung to the other.

It is only partial rupture of a hydatid cyst that would cause dissemination of the hydatid. Complete rupture would invariably lead to death, either due to anaphylactic shock or fatal embolism. After a partial rupture of a simple hydatid cyst in the lung, the scolices, which are disseminated, have the potentiality to grow into a cyst again. Deve (Barrett, 1960) proved that scolices could revert to cysts-a curious biological phenomenon known as 'Retrograde Metamorphism'. Scolices are the operating agents in their secondary spread (Barrett). Partial rupture may occur into the pleural cavity, which results in the formation of adhesions and a hydropneumothorax. If it ruptures in the lung parenchyma, the cysts subsequently spread into the adjacent areas. An endobronchial spread after a partial rupture will disseminate the cysts to different lobes and to the opposite side; this could also occur when it ruptures into a vein.

Multiple cysts with different sizes. Usually hydatid cysts implanted at the same time grow evenly and all the resulting cysts are of the same size. When they are of different sizes, either the infestation has taken place more than once, or one of the cysts is infected early during its growth and, therefore, remains small. If a coincident liver cyst exists, the lung cyst is invariably a metastasis from it, either through the diaphragm or through the blood route.

Intrabronchial spread of hydatid cysts. Whether intrabronchial dissemination of hydatid occurs or not is still a matter of controversy. Barrett (1960) states that intrabronchial spread probably never occurs in man. Deve (Barrett, 1960), however, has succeeded in producing bronchial dissemination in rats. I feel that its existence in man cannot be ruled out. The presence of multiple bilateral cysts of varying sizes situated peripherally (as seen in Case 1) strongly suggests the possibility of an intrabronchial spread. Also, in Case 2, with all the three cysts of different sizes and one of them infected, this possibility of intrabronchial spread cannot be ruled out. Further experience and careful research would alone enable us to find a suitable solution.

The experimental evidence, as Barrett (1960) mentions, suggests that the hexacanth embryo that settles in the lung and grows into a cyst has migrated in the blood vessels from the stomach and further through the capillary meshwork of the liver. But, by getting into the lymphatic channels of the stomach it could journey down by way of the thoracic duct and mediastinal lymphatics directly or through the superior vena cava to the lungs. Secondly, if live ova were introduced into the alimentary canal of laboratory animals, such as rabbits, monkeys or rats, the majority of the cysts that developed would be pulmonary. It is very difficult to prove this in man.

It has been postulated that hydatid disease in man is not comparable with that in animals. There are variations in behaviour of the parasite in men of different countries (Barrett, 1960), beside much individual variation in susceptability to hydatid infestation (Susman, 1953-quoted by Spencer, 1962). The study of these three patients strongly supports this view. Multiple pulmonary cysts in Australia are rare but occur in at least a quarter of the cases in England (Barrett, 1960).

\section{Summary}

Three cases with multiple pulmonary hydatid cysts are reported where the number of cysts encountered was three or more. This unusual occurrence is thought to be due to a probable intrabronchial spread. In one of the cases not only was the association of liver and lung hydatid seen. but there were also two separate cysts in the liver. The occurrence of two independent liver cysts is probably due to either a partial rupture of a simple cyst, or repeated infestation on different occasions.

Obliteration of the cyst cavity is an important technical step to prevent the problem of dead space infection and broncho-pulmonary fistulae. It also enables the surrounding lung to expand well. The prognosis with multiple hydatid cysts in the lungs is very good. The striking absence of recurrence of cysts, in spite of their rupture during removal could be due to the effective sterilization by $5 \%$ formalin.

This report is intended to demonstrate the interesting and unusual behaviour of the Echinococcus granulosus in India.

\section{Acknowledgments}

My grateful thanks are due to Dr P. L. Power, M.D., F.C.P.S., the Dean of the Medical College and Hospital of Nagpur, India, for allowing me to publish the hospital records, to Professor A. L. d'Abreu, O.B.E., CH.M., F.R.C.S., professor of Surgery of the Queen Elizabeth Hospital, Birmingham, and $\mathrm{Mr} \mathrm{D}$. B. Clarke, F.R.C.S., for their valuable guidance, and to $\mathrm{Mr}$ Joshirao for the photographs.

\section{References}

Anderson, G.S. \& Brown, D.A.P. (1959-60) A case of hydatid cyst of the pancreas. Brit. J. Surg. 47, 147. 
Barrett, N.R. (1960) Anatomy and pathology of multiple hydatid cysts in the thorax. Ann. roy. Coll. Surg. Engl. 26, 362.

Barrett, N.R. \& Thomas, D. (1952-53) Pulmonary hydatid disease. Brit. J. Surg. 40, 222.

BorRIE, J. (1962-63) Fifty thoracic hydatid cysts. Brit. J. Surg. 50, 268.

D'Abreu, A.L. (1938) Lobectomy for hydatid disease of the lung. J. int. Chir. (Personal communication).

D'ABreU, A.L. (1950) The removal of a hydatid cyst from the wall of the left ventricle. Thorax, 5, 362 .

D'Abreu, A.L. \& LAmbert Rogers (1938) Bilateral pulmonary hydatid cysts. Brit. J. Surg. (Personal Communication).
DEW, H.R. (1957-58) Morphological variation in hydatid disease. Brit. J. Surg. 45, 447.

Dick, E. (1962-63) A survey of hydatid disease in the Waikato Hospital Board area from 1942-59, Hamilton, New Zealand. Aust. N.Z. J. Surg. 32, 197.

EARLEY, T.K. \& Hunt, A.H. (1964) Hydatid disease of the liver (The treatment of intrabiliary hydatid with biliary fistula). Brit. J. Surg. 51, 50.

SPencer, H. (1962) Pathology of the excluding Lung Pulmonary Tuberculosis. Pergamon Press, Oxford.

Thомаs, D.M.E. (1965) Hydatid diseases of the lung. Clinical Surgery - Thorax (Ed. by Charles Rob and Rodney Smith), p. 210.

Toole, H., Propatoridis, J. \& Pangalos, N. (1953) Intrapulmonary rupture of hydatid cyst of the liver. Thorax, $\mathbf{8}$, 274. 